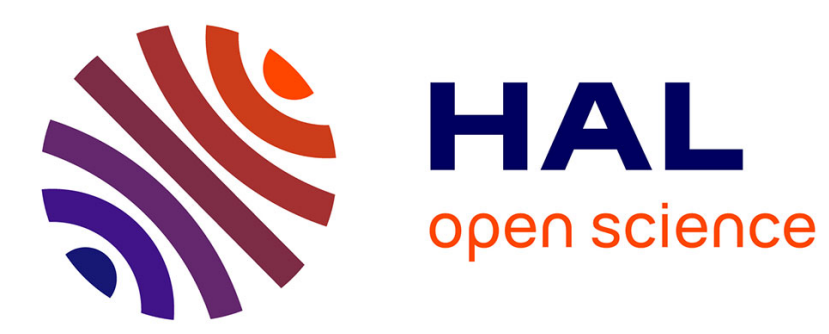

\title{
Analysis of inhomogeneous materials at large strains using fast Fourier transform
}

Noël Lahellec, Jean-Claude Michel, Hervé Moulinec, Pierre Suquet

\section{To cite this version:}

Noël Lahellec, Jean-Claude Michel, Hervé Moulinec, Pierre Suquet. Analysis of inhomogeneous materials at large strains using fast Fourier transform. Computational Mechanics of Solid Materials at large Strains, 2003, Stuttgart, 2001, Germany. pp.247-258. hal-00097030

\section{HAL Id: hal-00097030 \\ https://hal.science/hal-00097030}

Submitted on 8 Dec 2008

HAL is a multi-disciplinary open access archive for the deposit and dissemination of scientific research documents, whether they are published or not. The documents may come from teaching and research institutions in France or abroad, or from public or private research centers.
L'archive ouverte pluridisciplinaire HAL, est destinée au dépôt et à la diffusion de documents scientifiques de niveau recherche, publiés ou non, émanant des établissements d'enseignement et de recherche français ou étrangers, des laboratoires publics ou privés. 


\title{
ANALYSIS OF INHOMOGENEOUS MATERIALS AT LARGE STRAINS USING FAST FOURIER TRANSFORMS
}

\author{
N. Lahellec, J.C. Michel, H. Moulinec, P. Suquet \\ L.M.A./ C.N.R.S. \\ 31 Chemin Joseph Aiguier \\ 13402. Marseille. Cedex 20. France \\ suquet@ma.cnrs-mrs.fr
}

\section{Introduction}

Every material is naturally inhomogeneous at a small enough scale. The aim of homogenization is to replace a microscopically inhomogeneous material by a macroscopically homogeneous medium, the properties of which are the effective properties of the initial inhomogeneous material. However, nonlinear phenomenas such as fatigue or rupture are often triggered by local stress or strain concentrations at small scale. Therefore, besides effective properties, it is also essential to investigate how local fields are infl uenced by, and infl uence in turn, the microstructure of a material.

Precise determination or accurate estimation of local fields may involve considerable computational efforts. Direct numerical simulations of composit es with periodic microstructures have been performed by the Finite Element Method (FEM) since the early seventies, but despite numerous achievements, the application of this method to three-dimensional problems is still limited by the complexity of meshes. The need for investigating materials with complex microstructure without meshing provided a first motivation for developing a new computational method, specifically devised for microstructures, which could make direct use of images of microstructures, as delivered by Scanning Electron Microscopy or Computed Tomography ([1] [2] [3]).

A second motivation for developing a method capable of simulating easily the response of different microstructures stems from the recent theoretical models of the effective propert ies of nonlinear composites (see the review article Ponte Castañeda and Suquet [4] and the references herein). Direct comparison of these models with experimental data is often difficult, in that many different 
phenomena may occur simultaneously in the nonlinear regime (plasticity but also damage, interphase debonding, grain boundary sliding...). A comparison with numerical simulations, although only a step towards a complete validation, permits to ăctivate’all nonlinear mechanisms independently.

\section{Solving a periodic elasticity problem with the FFT}

As a reminder for readers who are not familiar with the FFT method, the case of linear elasticity at small strains is discussed first. The microstruct ure of the composite material is described by a representative volume element (r.v.e.) $V$ comprised of $N$ homogeneous phases. The $N$ individual constituents are linear elastic, with stiffness $\boldsymbol{c}(\boldsymbol{x})$ and perfectly bonded accross their interfaces. The volume element is subjected to an average strain $\boldsymbol{E}$ and the local problem to be solved for the local stress and strain fields reads as

$$
\boldsymbol{\sigma}(\boldsymbol{x})=\boldsymbol{c}(\boldsymbol{x}): \boldsymbol{\varepsilon}(\boldsymbol{u}(\boldsymbol{x})), \operatorname{div}(\boldsymbol{\sigma}(\boldsymbol{x}))=\mathbf{0}, \quad\langle\boldsymbol{\varepsilon}\rangle=\boldsymbol{E},
$$

where $\langle$.$\rangle denotes the spatial average over V$. The bound ary conditions applied on $\partial V$ should reproduce as closely as possible the in situ state of the r.v.e. This state is very seldomly known and periodicity conditions are assumed to close the problem : the local strain field is decomposed into its average and a periodic fluctuation $\varepsilon(\boldsymbol{u}(\boldsymbol{x}))=\boldsymbol{E}+\boldsymbol{\varepsilon}\left(\boldsymbol{u}^{*}(\boldsymbol{x})\right.$ ) where $\boldsymbol{u}^{*}$ is periodic (not ation $\left.\boldsymbol{u}^{*} \#\right)$. The tensions $\boldsymbol{\sigma} . \boldsymbol{n}$ take opposite values on oppposite sides of the r.v.e. (not ation $\boldsymbol{\sigma} . \boldsymbol{n}-\#$ ). The reader is referred to Suquet [5] for more details about periodicity conditions.

The local problem (1) can be re-written by introducing a homogeneous reference material with elastic stiffness $c^{0}$ :

$$
\boldsymbol{\sigma}(\boldsymbol{x})=\boldsymbol{c}^{0}: \boldsymbol{\varepsilon}\left(\boldsymbol{u}^{*}(\boldsymbol{x})\right)+\boldsymbol{\tau}(\boldsymbol{x}), \operatorname{div}(\boldsymbol{\sigma}(\boldsymbol{x}))=\mathbf{0}, \quad \boldsymbol{u}^{*} \#, \boldsymbol{\sigma} \cdot \mathbf{n}-\#,
$$

where the polarization field $\boldsymbol{\tau}(\boldsymbol{x})$ is given by :

$$
\boldsymbol{\tau}(\boldsymbol{x})=\boldsymbol{\delta} \boldsymbol{c}(\boldsymbol{x}):\left(\varepsilon\left(\boldsymbol{u}^{*}(\boldsymbol{x})\right)+\boldsymbol{E}\right)+\boldsymbol{c}^{0}: \boldsymbol{E}, \quad \boldsymbol{\delta} \boldsymbol{c}(\boldsymbol{x})=\boldsymbol{c}(\boldsymbol{x})-\boldsymbol{c}^{0} .
$$

The solution of problem (2) can be expressed by means of the periodic Green's operator associated with the elasticity tensor $c^{0}$ and reads, in real space and Fourier space respectively :

$$
\varepsilon\left(\boldsymbol{u}^{*}(\boldsymbol{x})\right)=-\boldsymbol{\Gamma}^{0} * \boldsymbol{\tau}(\boldsymbol{x}), \quad \hat{\boldsymbol{\varepsilon}}^{*}(\boldsymbol{\xi})=-\hat{\boldsymbol{\Gamma}}^{0}(\boldsymbol{\xi}): \hat{\boldsymbol{\tau}}(\boldsymbol{\xi}) \forall \boldsymbol{\xi} \neq \mathbf{0}, \quad \hat{\varepsilon}^{*}(\mathbf{0})=\mathbf{0} .
$$

After substituting back the expression (3) of $\tau$ in this relation, the initial local problem (1) reduces to the periodic Lippmann-Schwinger integral equation for $\varepsilon(\boldsymbol{u})$ which reads, in real space:

$$
\boldsymbol{\varepsilon}(\boldsymbol{u}(\boldsymbol{x}))=-\boldsymbol{\Gamma}^{0} *(\boldsymbol{\delta} \boldsymbol{c}(\boldsymbol{x}): \boldsymbol{\varepsilon}(\boldsymbol{u}(\boldsymbol{x})))+\boldsymbol{E} .
$$


The operat or $\Gamma^{0}$ is explicitely known in Fourier space for arbitrary anisotropy of the reference medium:

$$
\hat{\Gamma}_{i j k h}^{0}=\left.N_{i k}^{0} \xi_{j} \xi_{h}\right|_{(i j k h)}, \quad K_{i k}^{0}=c_{i j k h}^{0} \xi_{j} \xi_{h}, \quad \boldsymbol{N}^{0}=\left(\boldsymbol{K}^{0}\right)^{-1},
$$

where the symbol ${ }_{(i j k h)}$ denotes symmetrization with respect to the four indices $(i j k h)$ resulting in min or and major symmetries for $\hat{\boldsymbol{\Gamma}}^{0}$. Specific expressions for $\Gamma^{0}$ can be found in Mura [6] for different classes of anisotropy of the reference medium.

The integral equation (4) is solved by a fixed-point method :

$$
\varepsilon\left(\boldsymbol{u}^{\mathrm{i}+1}\right)=-\boldsymbol{\Gamma}^{0} *\left(\boldsymbol{\delta} \boldsymbol{c}: \boldsymbol{\varepsilon}\left(\boldsymbol{u}^{\mathrm{i}}\right)\right)+\boldsymbol{E} .
$$

This algorithm can be further simplified by noting that $\Gamma^{0} *\left(\boldsymbol{c}^{0}: \varepsilon\right)=\varepsilon\left(\boldsymbol{u}^{*}\right)$ and the iterative method reads

$$
\varepsilon\left(\boldsymbol{u}^{\mathrm{i}+1}\right)=\varepsilon\left(\boldsymbol{u}^{\mathrm{i}}\right)-\Gamma^{0} * \boldsymbol{\sigma}^{\mathrm{i}}, \quad \boldsymbol{\sigma}^{\mathrm{i}}(\boldsymbol{x})=\boldsymbol{c}(\boldsymbol{x}): \varepsilon\left(\boldsymbol{u}^{\mathrm{i}}(\boldsymbol{x})\right) .
$$

The $\mathrm{i}+1$-th iterate of the numerical algorithm typically reads :

$$
\begin{array}{ll} 
& \boldsymbol{\varepsilon}^{\mathrm{i}} \text { and } \boldsymbol{\sigma}^{\mathrm{i}} \text { being known } \\
\text { a) } & \hat{\boldsymbol{\sigma}}^{\mathrm{i}}=\mathrm{FT}\left(\boldsymbol{\sigma}^{\mathrm{i}}\right), \\
\text { b) } & \text { Convergence test } \\
\text { c) } & \hat{\boldsymbol{\varepsilon}}^{\mathrm{i}+1}(\boldsymbol{\xi})=\hat{\boldsymbol{\varepsilon}}^{\mathrm{i}}(\boldsymbol{\xi})-\hat{\boldsymbol{\Gamma}}^{0}(\boldsymbol{\xi}): \hat{\boldsymbol{\sigma}}^{\mathrm{i}}(\boldsymbol{\xi}) \forall \boldsymbol{\xi} \neq \mathbf{0} \text { and } \hat{\boldsymbol{\varepsilon}}^{\mathrm{i}+1}(\mathbf{0})=\boldsymbol{E}, \\
\text { d) } & \boldsymbol{\varepsilon}^{\mathrm{i}+1}=\mathrm{FT}^{-1}\left(\hat{\boldsymbol{\varepsilon}}^{\mathrm{i}+1}\right) \\
\text { e) } & \boldsymbol{\sigma}^{\mathrm{i}+1}(\boldsymbol{x})=\boldsymbol{c}(\boldsymbol{x}): \boldsymbol{\varepsilon}^{\mathrm{i}+1}(\boldsymbol{x}), \quad \forall \boldsymbol{x} \in V .
\end{array}
$$

FT stands for the Fourier transform. Convergence is reached when $\sigma^{i+1}$ is in equilibrium.

In practice the microstructure is given in the form of an image, consisting of pixels (or voxels in dimension three) of a given size. The spatial resolution of the image is the number of pixels along each coordinate axis. The image sampling in real space generates a corresponding sampling in Fourier space. The algorithm (8) is implemented in discretized form, the FT being replaced by the Fast Fourier Transform (FFT) at tached to the above sampling in real and Fourier space ([2]).

The FFT method can be used in most problems where the FEM is used, provided that periodicity conditions can be adopted for the problem at hand. Extensions of the method to plasticity with or without hardening, to viscoplasticity, to phase transformation were given in [2] [7] [8] in the context of infinit esimal strains. The next sections are concerned with the extension of the method to large strains. 


\section{Hyperelastic materials}

Consider now the case where the deformations applied to the composite material are large. The shape of the r.v.e. $V$ evolves with time and classically two configurations play a particular role, the initial configuration $V_{0}$ and the current configuration $V(t)$. When the individual constituents are hyperelastic a Lagrangian formulation can be adopted and the local problem can be posed on the initial configuration $V_{0}$ only. A particle which was initially at point $\boldsymbol{X}$ moves to a location $\boldsymbol{x}=\boldsymbol{x}(\boldsymbol{X}, t)=\boldsymbol{X}+\boldsymbol{u}(\boldsymbol{X}, t)$ at time $t$. The deformation gradient and the first Piola-Kirchhoff stress tensor $\boldsymbol{\pi}$ are related by:

$$
\boldsymbol{f}=\boldsymbol{I}+\nabla_{\boldsymbol{X}} \boldsymbol{u}(\boldsymbol{X}, t), \quad \boldsymbol{\pi}(\boldsymbol{X})=\frac{\partial w}{\partial \boldsymbol{f}}(\boldsymbol{X}, \boldsymbol{f}),
$$

where $w(\boldsymbol{X},$.$) is the strain energy at point \boldsymbol{X}$. The average gradient $\boldsymbol{F}$ of the transformation is applied incrementally along a prescribed path in the loading space. This path is parametrized by a scalar variable $t$. The local problem to be solved reads :

$$
\left.\begin{array}{c}
\boldsymbol{\pi}(\boldsymbol{X})=\frac{\partial w}{\partial \boldsymbol{f}}(\boldsymbol{X}, \boldsymbol{f}), \quad \operatorname{div}_{\boldsymbol{X}} \boldsymbol{\pi}=\mathbf{0} \text { in } V_{0}, \\
\boldsymbol{f}(\boldsymbol{X})=\boldsymbol{F}+\nabla_{\boldsymbol{X}} \boldsymbol{u}^{*}, \quad \boldsymbol{u}^{*} \#, \boldsymbol{\pi} \cdot \boldsymbol{N}-\# \text { on } \partial V_{0}
\end{array}\right\}
$$

where $\boldsymbol{N}$ denotes the outer normal unit vector to $\partial V_{0}$.

The local problem (10) is similar to (2). It is posed on a fixed configuration which allows us to use the FFT method with fixed grids in real space and Fourier space. The constitutive relations are nonlinear and the whole gradient $f$ (and not only it s symmetric part) are taken into account into (10). The problem (10) is solved step-by-step in time, with a Newton-Raphson algorithm at each time step.

\subsection{Newton-Raphson algorithm}

$\boldsymbol{F}_{t+\Delta t}$ is imposed at time $t+\Delta t$. The principal unknown is the gradient $\nabla \boldsymbol{u}_{t+\Delta t}^{*}$ of the periodic fluctuation of the displacement. This gradient is determined by imposing that $\boldsymbol{\pi}_{t+\Delta t}$ is in equilibrium.

Let $\boldsymbol{\pi}^{i}, \boldsymbol{f}^{i}$ and $\left(\boldsymbol{u}^{*}\right)^{i}$ denote the iterates approximating $\boldsymbol{\pi}_{t+\Delta t}, \boldsymbol{f}_{t+\Delta t}$ and $\boldsymbol{u}_{t+\Delta t}^{*}$.

Iterate $i: \nabla\left(\boldsymbol{u}^{*}\right)^{i-1}$ being known :

(1) Compute $\boldsymbol{f}^{i-1}$ and $\boldsymbol{\pi}^{i-1}$

$$
\boldsymbol{f}^{i-1}(\boldsymbol{X})=\boldsymbol{F}_{t+\Delta t}+\nabla\left(\boldsymbol{u}^{*}\right)^{i-1}(\boldsymbol{X}), \boldsymbol{\pi}^{i-1}(\boldsymbol{X})=\frac{\partial w}{\partial \boldsymbol{f}}\left(\boldsymbol{X}, \boldsymbol{f}^{i-1}(\boldsymbol{X})\right) .
$$


Check if $\boldsymbol{\pi}^{i-1}$ is in equilibrium. If not, then

(2) Solve the linear tangent problem for the periodic field $\nabla\left(\delta \boldsymbol{u}^{*}\right)$

$$
\left\langle\frac{\partial^{2} w}{\partial \boldsymbol{f}^{2}}\left(\boldsymbol{f}^{i-1}\right): \nabla\left(\delta \boldsymbol{u}^{*}\right): \nabla \boldsymbol{v}\right\rangle=-\left\langle\boldsymbol{\pi}^{i-1}: \nabla \boldsymbol{v}\right\rangle \quad \forall \boldsymbol{v} \#
$$

(3) Update $\nabla\left(\boldsymbol{u}^{*}\right)^{i}: \nabla\left(\boldsymbol{u}^{*}\right)^{i}(\boldsymbol{X})=\nabla\left(\boldsymbol{u}^{*}\right)^{i-1}(\boldsymbol{X})+\nabla\left(\delta \boldsymbol{u}^{*}\right)(\boldsymbol{X})$.

The criterion which serves to check equilibrium reads :

$$
\max _{\boldsymbol{\xi}}\left|\boldsymbol{\xi} \cdot \widehat{\boldsymbol{\pi}}^{i}(\boldsymbol{\xi})\right| \leq \varepsilon\left|\widehat{\boldsymbol{\pi}}^{i}(\mathbf{0})\right|, \quad \text { where typically } \varepsilon=10^{-4} .
$$

The problem (12) is solved iteratively using the FFT method described in section 2. More specifically an internal loop is performed to find $\nabla\left(\delta \boldsymbol{u}^{*}\right)(\boldsymbol{X})$

$$
\nabla\left(\delta \boldsymbol{u}^{*}\right)^{k}=\nabla\left(\delta \boldsymbol{u}^{*}\right)^{k-1}-\tilde{\boldsymbol{\Gamma}}^{0} *\left(\boldsymbol{L}^{i-1}: \nabla\left(\delta \boldsymbol{u}^{*}\right)^{k-1}+\boldsymbol{\pi}^{i-1}\right),
$$

where $\boldsymbol{L}^{i-1}(\boldsymbol{X})=\frac{\partial^{2} w}{\partial \boldsymbol{f}^{2}}\left(\boldsymbol{X}, \boldsymbol{f}^{i-1}(\boldsymbol{X})\right) \cdot \tilde{\boldsymbol{\Gamma}}^{0}$ is defined in Fourier space as

$$
\hat{\tilde{\Gamma}}_{i j k h}^{0}=\left.N_{i k}^{0} \xi_{j} \xi_{h}\right|_{(i k)(j h)},
$$

where the symbol ${ }_{(i k)(j h)}$ denotes symmetrization with respect to the indices $i, k$ and $j, h$ only.

\subsection{Example: fiber-reinforced elastomer}

The above algorithm has been applied to model the deformation of an elastomeric matrix reinforced by stiff fibers. In its initial configuration the unit cell was a square cont aining 64 circular identical impenetrable fibers, with $25 \%$ volume fraction, arranged randomly in the unit cell. The matrix and the fibers were compressible Mooney-Rivlin materials with strain energy :

$$
w(\boldsymbol{f})=\frac{\mu}{2}\left(i_{1}-3\right)-\mu \ln j+\frac{\kappa}{2}(j-1)^{2}, i_{1}=\operatorname{tr}\left({ }^{\top} \boldsymbol{f} \cdot \boldsymbol{f}\right), j=\operatorname{det} \boldsymbol{f} .
$$

The fibers were 10 times stiffer than the matrix. 10 different configurations were tested. The macroscopic deformation was a biaxial isochoric deformation $\boldsymbol{F}=\exp \left(E_{1}\right) \boldsymbol{e}_{1} \otimes \boldsymbol{e}_{1}+\exp \left(-E_{1}\right) \boldsymbol{e}_{2} \otimes \boldsymbol{e}_{2}+\boldsymbol{e}_{3} \otimes \boldsymbol{e}_{3}$, where the logarithmic strain in the first direction $E_{1}$ was negative (contraction in direction 1, extension in direction 2).

The deformed states of a typical configuration are shown in figure 1. The stress-strain response of all 10 different configurations are shown in Figure 

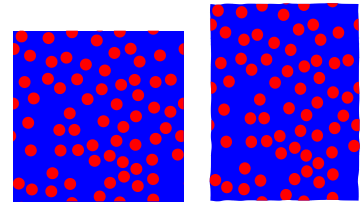

$E_{1}=0$

$E_{1}=-0.14$

(b)

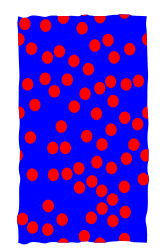

$E_{1}=-0.28$
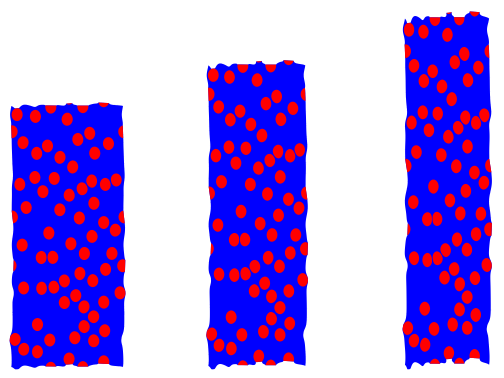

$E_{1}=-0.42$

$E_{1}=-0.56$

$E_{1}=-0.70$

$(d)$

$(e)$

$(f)$

Figure 1. Hyperelastic matrix reinforced by 64 circular fibers. (a): initial configuration. (b)-(f): successive deformed states for different values of the macroscopic logarithmic strain $E_{1}=\ln F_{11}$.

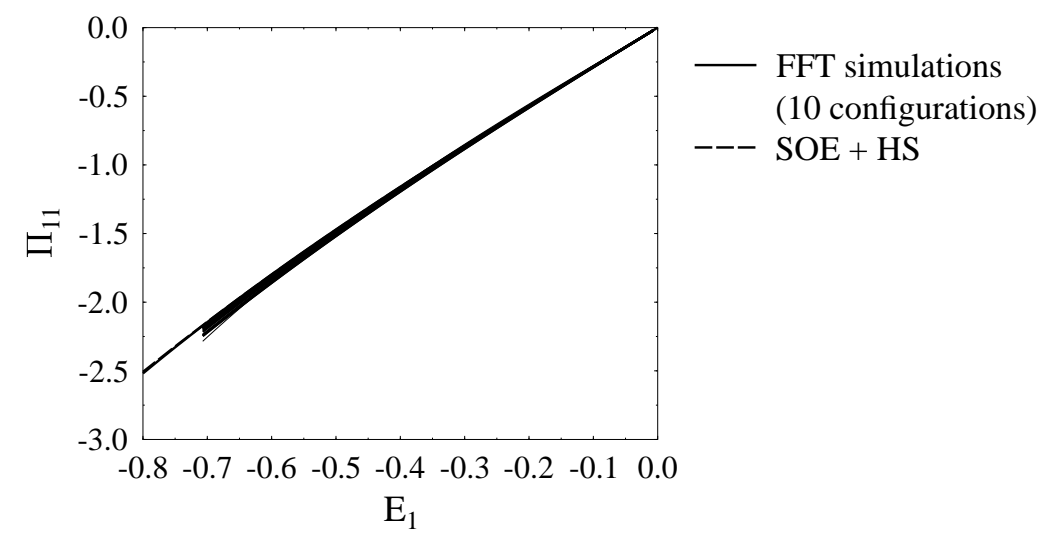

Figure 2. Macroscopic stress-strain curves. First Piola-Kirchhoff stress as a function of the logarithmic strain in direction 1. 10 different configurations. Comparison between the FFT simulations and the second order estimate (SOE) with the lower Hashin-Shtrikman estimate. 
2. They are seen to be very close to one another. Also shown in Figure 2 is the prediction of the second-order estimate (SOE) of Ponte Castañeda [9] implemented with the Hashin-Shtrikman estimate (lower bound) for circular fibers in a matrix. As can be seen from this figure, the agreement between the theory and the numerical calculations is excellent.

\section{Eulerian formulation}

\subsection{Local problem}

We now turn to the case where the constitutive relations of the constituents can be expressed by means of quantities defined on the current configuration only, the Cauchy stress $\boldsymbol{\sigma}$ and the Eulerian strain-rate $\boldsymbol{d}$ for instance. This is the case for Newt onian fl uids for which the constitutive relations read

$$
\boldsymbol{\sigma}(\boldsymbol{x})=\boldsymbol{L}(\boldsymbol{x}): \boldsymbol{d}(\boldsymbol{x}),
$$

and more generally for viscous materials when elasticity effects are neglected (the case of power-law viscous materials is considered in Lebensohn [10]). For simplicity we restrict our attention to Newtonian fl uids as described by (15).

The geometry of the r.v.e. evolves with time in two ways. First the unit vectors $\boldsymbol{Y}_{i}$ defining the unit cell are convected by the macroscopic velocity gradient:

$$
\dot{\boldsymbol{Y}}_{i}(t)=\langle\operatorname{grad} \boldsymbol{v}(t)\rangle . \boldsymbol{Y}_{i}(t) .
$$

Second, the position of the phases within the r.v.e. changes with time, the material particles being convected by the microscopic velocity field:

$$
\dot{\boldsymbol{x}}(t)=\boldsymbol{v}(\boldsymbol{x}, t)
$$

The local problem to be solved in the Eulerian configuration consists of the constitutive relations, together with the equilibrium and compatibility equations. At each time $t$ the local problem on the current configuration can be solved using the FFT method described in section 2. It remains to see how the unit cell changes with time.

\subsection{A Multi-Particle Method}

The two grids. The proposed method falls in the category of multi-particle methods and follows rather closely the Particle-In-Cell (PIC) method proposed by Sulsky, Chen and Schreyer [11, 12], with a difference stemming from the updating of the computational grid (the method proposed by Lebensohn [10] is also similar in spirit). The underlying idea of the PIC method is to consider two separate grids: 
- The computational grid is used for applying the FFT method described in section 2. It is a regular grid but it does not have to be rectangular (Fourier transforms can be defined on noncubic lattices).

- The material grid is attached to the material particles. It does not have to be structured or regular and can be seen as a collection of particles, rather than as a structured mesh. It is only used to apply the constitutive relations (which are material relations). In the FFT method it is used in real space only. In most methods the material grid is finer than the computational one (by a fact or of 4 to 9 ).

Each grid carries its own set of unknowns. In other words,

- the computational grid carries unknowns labelled with lowerscript $c$ (as in 'computations) : $\boldsymbol{x}_{c}^{(i, j)}=(i-1) \boldsymbol{Y}_{1}+(j-1) \boldsymbol{Y}_{2}$ denote the nodes of the computational grid, $\boldsymbol{\sigma}_{c}, \boldsymbol{v}_{c}$ denote the stress and velocity fields at these nodes.

- the material grid carries unknowns labelled with lowerscript $p$ (as in particles): $\boldsymbol{x}_{p}$ denote the location of the particles, $\boldsymbol{\sigma}_{p}, \boldsymbol{v}_{p}$ denote the stress and velocity field at the particles.
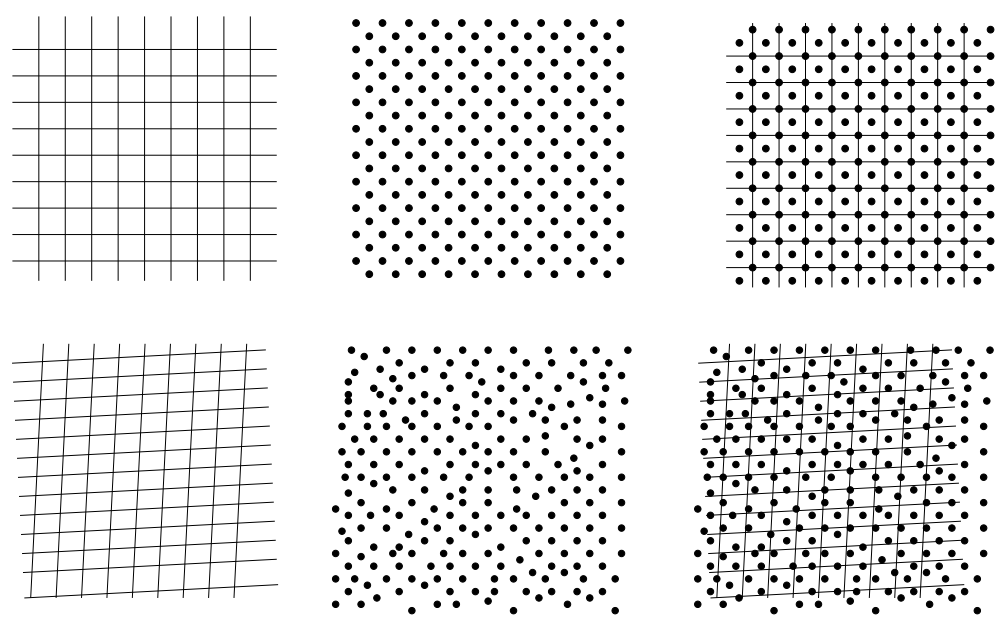

Figure 3. Top row: initial grids. Left: the computational grid. Center: the material grid. Right: both grids superimposed. Bottom row: convected grids.

\subsection{Updating the grids}

In the initial PIC method ([11, 12]), the computational grid is fixed. However in the problem under consideration here the computational grid is associated 
with the unit cell which generates the whole microstructure by periodicity and cannot remain fixed. The unit vectors $\boldsymbol{Y}_{i}$ defining the periodic lattice are material vectors and follow the macroscopic velocity field according to (16). The computational grid is updated accordingly. The material grid is updated using the microsocopic velocity field according to (17).

The grids are updated using an explicit scheme. At time $t_{n}$, the velocity fields $\boldsymbol{v}_{c}\left(t_{n}\right)$ is computed on the computational grid and transferred (see below) onto the material grid to get a field $\boldsymbol{v}_{p}\left(t_{n}\right)$. Then :

$$
\left.\begin{array}{c}
\boldsymbol{Y}_{i}\left(t_{n+1}\right)=\boldsymbol{Y}_{i}\left(t_{n}\right)+\Delta t\left\langle\operatorname{grad} \boldsymbol{v}_{c}\left(t_{n}\right)\right\rangle ._{i}(t), \\
\boldsymbol{x}_{c}\left(t_{n+1}\right)=(i-1) \boldsymbol{Y}_{1}\left(t_{n+1}\right)+(j-1) \boldsymbol{Y}_{2}\left(t_{n+1}\right), \\
\boldsymbol{x}_{p}\left(t_{n+1}\right)=\boldsymbol{x}_{p}\left(t_{n}\right)+\Delta t \boldsymbol{v}_{p}\left(\boldsymbol{x}_{p}\left(t_{n}\right)\right) .
\end{array}\right\}
$$

Transfer operators. Transfer operators are needed in order to transfer information from the computational grid to the material grid and vice-versa. Devising consistent transfer operators is certainly a crucial part of the algorithm.

Consider a computational grid with an initially rectangular pattern. In the initial configuration the grid nodes have coordinates $\left((i-1) \Delta x_{0},(j-1) \Delta y_{0}\right)$. In the current configuration the grid nodes have coordinates $\left(x_{i, j}^{c}, y_{i, j}^{c}\right)$. Let us introduce a family of shape functions $N^{(i, j)}, i=1, \ldots, I, j=1, \ldots, J$ (as is classical in the FEM) on the computational grid, where $I$ and $J$ denote the number of sampling points on the horizont al and vertical axis (for the initial grid) respectively. Given the regular geometry of the computational grid a natural choice for the $N^{(i, j)}$ are the interpolation functions of the 4 point quadrilateral element in the FEM (these functions were used in the example presented in section 4.4). Then, given the values $f_{c}^{(i, j)}=f\left(\boldsymbol{x}_{c}(i, j)\right.$ of any function $f$ at the computational nodes, the interpolated field at any other point $\boldsymbol{x}$ read as

$$
f(\boldsymbol{x})=\sum_{(i, j)=(1,1)}^{I, J} N^{(i, j)}(\boldsymbol{x}) f_{c}^{(i, j)} .
$$

These relations apply in particular at the particle positions $\boldsymbol{x}_{p}$.

The inverse transfer from the material grid to the computational grid is performed using the same interpolation functions. Each particle is assigned a mass $m_{p}$ and the mass of a computational node is defined as

$$
m\left(\boldsymbol{x}_{c}^{(i, j)}\right)=\sum_{p=1}^{P} m_{p} N^{(i, j)}\left(\boldsymbol{x}_{p}\right)
$$

Then the values $f_{c}$ of $f$ at any computational node $\boldsymbol{x}_{c}$ is given by :

$$
f_{c}\left(\boldsymbol{x}_{c}^{(i, j)}\right)=\frac{1}{m\left(\boldsymbol{x}_{c}^{(i, j)}\right)} \sum_{p=1}^{P} m_{p} N^{(i, j)}\left(\boldsymbol{x}_{p}\right) f_{p}\left(\boldsymbol{x}_{p}\right)
$$




\subsection{A test example}

To check the accuracy of the updat ing scheme, the problem of a rigid particle rotating in a shear fl ow of a newtonian fl uid with uniform and constant viscosity has been investigated. The fl uid is subjected to a macroscopic fl ow with strainrate $\boldsymbol{D}$ and rotation-rate $\boldsymbol{\Omega}$. Inertia effects are neglected. The solution (rot ation of the part icle) for a spheroidal particle with infinit esimal volume fraction $c^{(p)}$ in an incompressible newt onian fl uid goes back to Jeffery [13]. A generalization of Jeffery's result, also applying to compressible fl uids and viscous particles, can also be derived by means of Eshelby's result for ellipsoidal inclusions. This derivation will not be given here. For a rigid particle the evolution equation for a unit normal vect or $\boldsymbol{u}$ attached to the particle is

$$
\frac{d \boldsymbol{u}}{d t}=\boldsymbol{\omega}^{I} \cdot \boldsymbol{u}, \quad \boldsymbol{\omega}^{I}=\boldsymbol{\Omega}-\boldsymbol{\Pi}: \boldsymbol{S}^{-1}: \boldsymbol{D}
$$

where $S$ and $\Pi$ are the two Eshelby tensors of the particle in the matrix giving respectively the deformation-rate in the inclusion and to the rotation-rate of the inclusion.

This general three-dimensional solution can be specialized to dimension 2 . The result is

$$
\frac{d \boldsymbol{u}}{d t}=\boldsymbol{\Omega} \cdot \boldsymbol{u}+B(\boldsymbol{D} \cdot \boldsymbol{u}-(\boldsymbol{u} \cdot \boldsymbol{D} \cdot \boldsymbol{u}) \boldsymbol{u}),
$$

with $B=\left(r^{2}-1\right) /\left(r^{2}+1+2 r \frac{1-2 \nu}{2(1-\nu)}\right)$ where $r$ is the aspect-ratio of the particle and $\nu$ is the Poisson ratio of the linearly viscous matrix.
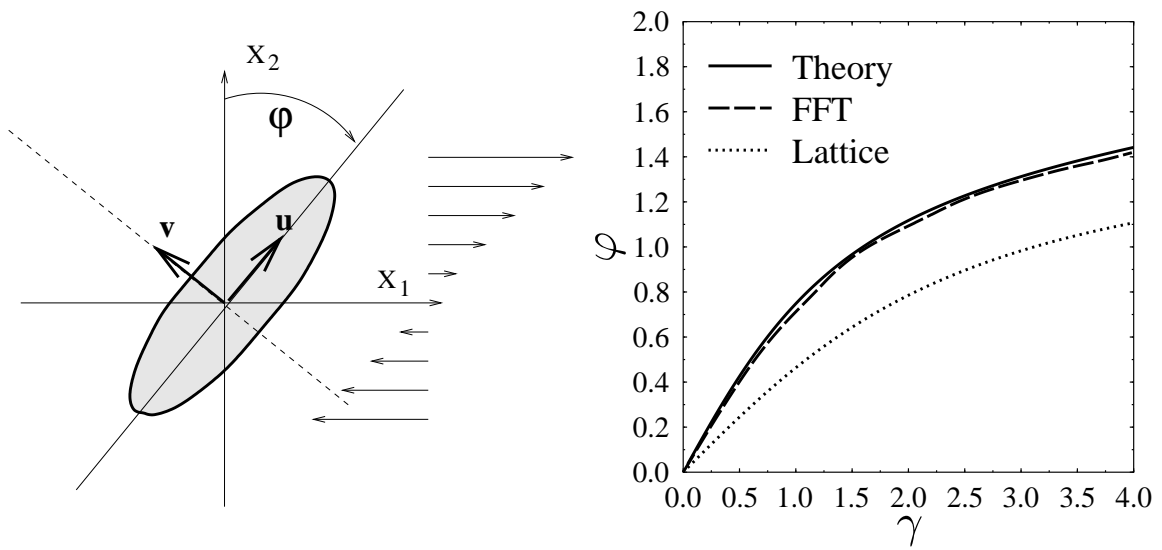

Figure 4. Particle rotating in a shear flow. 
In a shear flow,

$$
x_{1}(t)=X_{1}+\gamma(t) X_{2}, \quad x_{2}(t)=X_{2},
$$

(23) gives the the rotation of the unit normal vector $\boldsymbol{u}=\sin \varphi \boldsymbol{e}_{1}+\cos \varphi \boldsymbol{e}_{2}$ in the form :

$$
\frac{d \varphi}{d t}=\frac{\dot{\gamma}}{2}\left[1+B\left(\cos ^{2} \varphi-\sin ^{2} \varphi\right)\right]
$$

which can be integrated into

$$
\varphi(t)=\varphi(0)+\operatorname{Arctg}\left(R \operatorname{tg}\left(\frac{\gamma(t)}{R+1 / R}\right)\right), \quad \text { with } R=\sqrt{\frac{1+B}{1-B}} .
$$

A numerical simulation of the rotation of a particle in a shear flow has been conducted using the method described in section 4.2 with the following data

$$
r=3, \quad \nu=0.499, \quad c^{(p)}=0.3 \% .
$$

The comparison between the analytical and the numerical results is good as shown in Figure $4 \mathrm{~b}$. Also shown in this figure is the lattice rotation. As can be seen the particle rotates faster than the lattice.

\section{Concluding remarks}

Two ext ensions to finite strains of the numerical method based on Fast Fourier Transforms ([1, 2]) have been proposed and test examples have been analyzed. A few numerical issues deserve attention in fut ure work:

- In the Lagrangian approach presented in section 3, the rate of convergence of the it erative FFT algorithm applied to the tangent problem (13) can be rather poor. This is probably due to the fact that the tangent operator is only strongly elliptic (and not very strongly elliptic) and highly contrasted. An accelerated scheme would be very helpful.

- In the Eulerian approach presented in section 4, consistent transfer operators between the computational grid and the material grid are to be found.

\section{Acknowledgments}

The authors greatly benefited from discussions with R. Lebensohn, D. Sulsky and N. Triantafyllidis during the completion of this research. Part of it was carried out while Pierre Suquet was a Visiting Professor at Caltech. Wonderful interactions with K. Bhattacharya and M. Ortiz, financial support from Caltech and from the Délégation Générale à l'A rmement are grat efully acknowledged. 


\section{References}

[1] H. Moulinec and P. Suquet. A fast numerical method for computing the linear and nonlinear properties of composites. C. R. A cad. Sc. Paris II, 318:14171423, 1994.

[2] H. Moulinec and P. Suquet. A numerical method for computing the overall response of nonlinear composites with complex microstructure. Comp. Meth. Appl. Mech. Engng., 157:6994, 1998.

[3] J.C. Michel, H. Moulinec, and P. Suquet. Effect ive propert ies of composite mat erials with periodic microstruct ure: a comput ational approach. Comp. Meth. Appl. Mech. Engng., 172:109143, 1999.

[4] P. Ponte Castañeda and P. Suquet. Nonlinear composites. In E. Van der Giessen and T.Y. Wu, editors, Advances in Applied Mechanics, volume 34, pages 171302. Academic Press, New York, 1997.

[5] P. Suquet. Elements of Homogenization for Inelastic Solid Mechanics. In E. SanchezPalencia and A. Zaoui, editors, Homogenization Techniques for Composite Media, volume 272 of Lecture Notes in Physics, pages 193278, New York, 1987. Springer Verlag.

[6] T. MurA. Micromechanics of Defects in Solids. Martinus Nijhoff Publishers, Dordrecht, 1987.

[7] J.C. Michel, H. Moulinec, and P. Suquet. A computational method for linear and nonlinear composites with arbitrary phase contrast. Int. J. Numer. Meth. Engng, 52:139$160,2001$.

[8] W. Dreyer and W.H. Müller. A study of the coarsening in $\mathrm{Pb}$ / Sn solders. Int. J. Solids Structures, 37:38413871, 2000.

[9] P. Ponte Castañeda and E. Tiberio. A second-order homogenization method in finite elasticity and applications to black-filled elast omers. J. Mech. Phys. Solids, 48:1389_ $1411,2000$.

[10] R. Lebensohn. N-site modelling of a 3d viscoplastic polycrystal using Fast Fourier Transforms. Acta Materiala, 49:2723z737, 2001.

[11] D. Sulsky, Z. Chen, and H.L. Schreyer. A particle method for history-dependent materials. Comp. Methods A ppl. Mech. Engrg, 118:179196, 1994.

[12] D. Sulsky, S.J. Zhou, and H.L. Schreyer. Application of a particle-in-cell methods to solid mechanics. Computer Physics Communications, 87:236z52, 1995.

[13] G.B. Jeffery. The motion of ellipsoidal particles immersed in a viscous fluid. Proc. Royal Soc. London A, 102:161479, 1922. 